\title{
Crystallization Kinetics of Amorphous Alumina-Zirconia-Silica Ceramics
}

\author{
T. Chráska, * J. Hostomský, ** M. Klementová,** and J. Dubský* \\ * Institute of Plasma Physics of the ASCR, v.v.i., Za Slovankou 3, 18200 Prague, Czech Republic \\ ** Institute of Inorganic Chemistry of the ASCR, v.v.i., 25068 Husinec-Řež 1001, Czech Republic
}

Nanocrystalline ceramics has a wide potential of applications due to its significantly improved properties such as hardness, strength, abrasion resistance and toughness. Even though nowadays ceramic nanopowder can be conventionally synthesized in large quantities, the consolidation of nanoparticles into mesoscopic structures and large bulk pieces remains a challenge [1]. In the alternative approach such as plasma spraying, the powder compaction step is completely avoided by producing amorphous material by rapid solidification, which can then be thermally treated to introduce nanocrystalline structure by controlled crystallization [2].

Crystallization kinetics of amorphous alumina-zirconia-silica ceramics was studied for seven different material compositions (labeled as NZ, A, B, C, E, F in Fig. 1) based on the bulk cast ceramic material called Eucor ${ }^{\mathrm{TM}}$ [3]. The amorphous materials were prepared by plasma spraying thus the samples consist of thin splats with a significant chemical variation due to different proportions of $\mathrm{Al}$ and $\mathrm{Zr}$ oxides in the individual particles of injected powder. Each individual amorphous splat in the as-sprayed materials is, however, chemically homogeneous.

The amorphous materials crystallize above $920^{\circ} \mathrm{C}$ primarily as tetragonal $\mathrm{ZrO}_{2}$ solid solution with $\mathrm{Al}_{2} \mathrm{O}_{3}$ and to a small extent as $\delta-\mathrm{Al}_{2} \mathrm{O}_{3}$ in $\mathrm{Al}_{2} \mathrm{O}_{3}$-rich splats. The tetragonal zirconia nanocrystallites have average diameters increasing from 10 to $23 \mathrm{~nm}$ with increasing silica content (Fig. 2). For silica containing materials the nanocrystallites are embedded in silica rich remains of the amorphous matrix thus creating steep concentration gradients at the nanocrystal/glass interface. The kinetic stabilization of the nanoscale microstructure is related to the silica rejection during growth involving a soft impingement between neighboring nanocrystallites. Based on DSC measurements, the apparent activation energy of the crystallization increases approximately from 900 to $1500 \mathrm{~kJ} / \mathrm{mol}$ with decreasing silica content. According to the values of Avrami coefficient and on the microstructural observations, crystallization mechanism of the silica containing materials is best described by instantaneous nucleation followed by three dimensional crystal growth controlled by diffusion transport of $\mathrm{Zr}$ and $\mathrm{Si}$ cations in opposite directions (demixing). The Avrami exponent for the NZ material, however, points to a three dimensional growth mechanism controlled by a reaction at the phase boundary.

\section{References}

[1] M. Winterer, Springer Series in Materials Science (2002) 53.

[2] C. Suryanarayana, J. Miner. Met. Mater. Soc. (2002) 24.

[3] Eutit Ltd., www.eutit.cz.

[4] This work was carried out under the "Nanotechnologies for Society" program, supported by project no. KAN300430651 administered by the Academy of Sciences of the Czech Republic. 


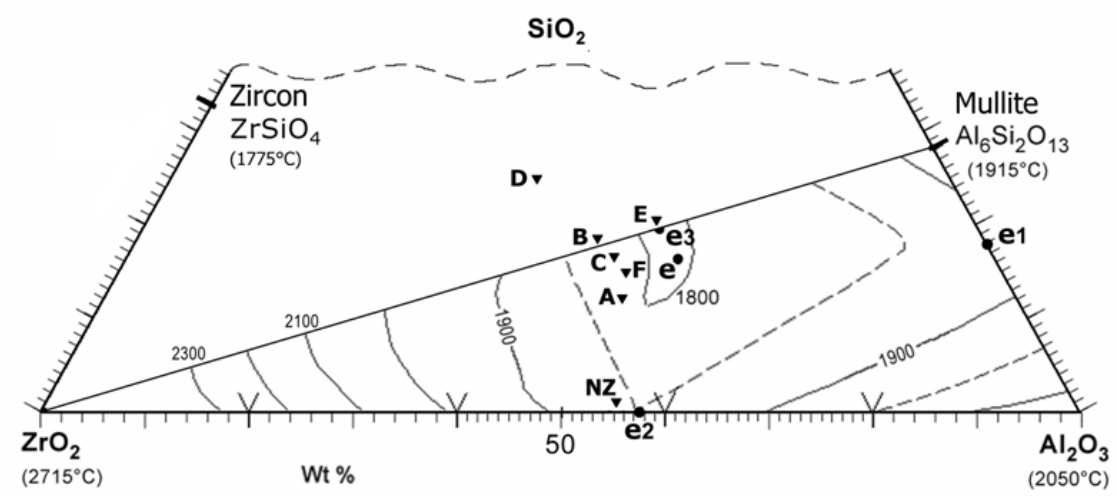

FIG. 1. Part of ternary equilibrium phase diagram showing different compositions of materials used in this study (marked by triangles). e1, e2, e3 - binary eutectic points, e - ternary eutectic point.
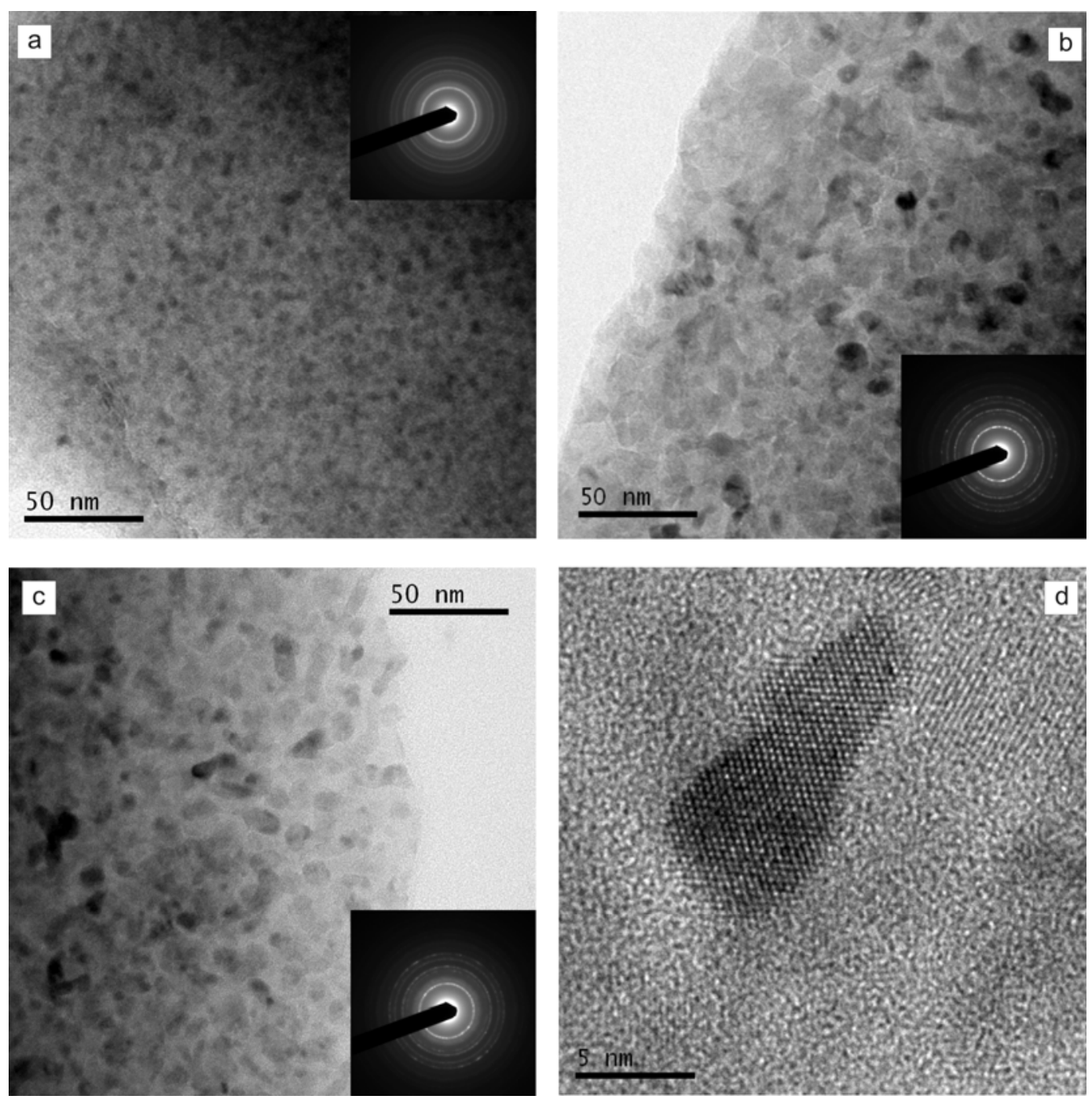

FIG. 2. Bright field TEM and HRTEM micrographs of annealed samples with corresponding diffraction patterns shown as insets. a) NZ, b) A, c) D, and d) a high resolution image of a tetragonal $\mathrm{ZrO}_{2}$ nanocrystal embedded in a weak contrast amorphous phase. 